\title{
NUMERICAL STUDIES OF DIFFERENT MIXED PHASE-FIELD FRACTURE MODELS FOR SIMULATING CRACK PROPAGATION IN PUNCTURED EPDM STRIPS
}

\author{
KATRIN MANG ${ }^{1}$ AND THOMAS WICK ${ }^{1}$ \\ ${ }^{1}$ Institute of Applied Mathematics, Leibniz Universität Hannover \\ Welfengarten 1, 30167 Hannover, mang@ifam.uni-hannover.de, wick@ifam.uni-hannover.de
}

Key words: Mixed Finite Elements, Phase-field Modeling, EPDM Rubber, Crack Propagation

\begin{abstract}
We consider a monolithic phase-field description for fractures in nearly incompressible materials, i.e., carbon black filled ethylene propylene diene monomer rubber (EPDM). A quasi-static phasefield fracture problem is formulated in mixed form based on three different energy functionals (AT2, AT1 and Wu's model) combined with two different stress splitting approaches (according to Miehe and Amor). It leads to six different phase-field fracture formulations in mixed form. The coupled variational inequality systems are solved in a quasi-monolithic manner with the help of a primal-dual active set method handling the inequality constraint. Further, adaptive mesh refinement is used to get a sharper crack zone. Numerical results based on the six different problem setups are validated on crack propagation experiments of punctured EPDM strips with five different test configurations. As a quantity of interest, the crack paths of experiments and numerical computations are discussed.
\end{abstract}

\section{INTRODUCTION}

The commonly used quasi-static phase-field fracture model in its classical displacement-based formulation fails due to locking effects if the considered solid is nearly incompressible. To allow simulating crack growth in rubber-like materials like EPDM rubber, this classical phase-field fracture model is extended to a mixed form of the solid-displacement equation resulting in two unknowns: a displacement field and a hydro-static pressure variable [14]. To fulfill an inf-sup condition, Taylor-Hood elements are employed for the displacement-pressure system. The fracture path is described with a phase-field function defined as a smoothed indicator variable. The crack irreversibility constraint is handled with a primal-dual active set method, see [11].

Considering finite element simulations of a test configuration with inclusion in the geometry as the proposed punctured EPDM strips, the impact of holes on the crack behavior is not fully understood in the literature. For example in [21], a pre-cracked sample with two holes is presented in a compressible solid and very different crack path results are shown for the same test. Further, the need for further studies on problems with holes is mentioned in [21, Section 8.2]. This led us to work in detail on results in EPDM with a circular hole in the specimen and computing numerical simulations based on the different energy functionals and stress splitting approaches. The main contributions of the work on hand are:

- Deriving stress splitting approaches along to Miehe et al. [15] and Amor et al. [4] for the elasticity equation in mixed form. 
- Formulating the incremental problem with three options for the energy functional along to Ambrosio Tortorelli [2, 3] (AT2 or AT1) or Wu [20].

- Computing five test configurations in punctured EPDM strips based on six different model problem formulations and comparing the crack paths.

The model problem formulation is given in Section 2. In Section 3, the results from conducted experiments with five test configurations are presented. Numerical results to compare the computed crack paths based on six different problem formulations and the experimental results are discussed in Section 4.

\section{PHASE-FIELD FRACTURE IN MIXED FORM}

We consider a two-dimensional, open and smooth domain $\Omega \subset \mathbb{R}^{2}$ with boundary $\Gamma_{D}$ on which Dirichlet conditions are prescribed. Let $I$ be a loading interval $[0, T]$, where $T>0$ is the end time value. A displacement function $u$ is given as $u:(\Omega \times I) \rightarrow \mathbb{R}^{2}$. Further, a smooth indicator function named phasefield is defined as $\varphi:(\Omega \times I) \rightarrow[0,1]$ with $\varphi=0$ broken and $\varphi=1$ unbroken. Assuming naturally a non-healing crack, irreversibility of the fracture has to be given, i.e., $\varphi$ has to be monotone non-increasing with respect to $t \in I$. Further, the $L^{2}$ scalar-product is denoted as $(a, b):=\int_{\Omega} a \cdot b d x$ for vectors $a, b$. The Frobenius scalar product of two matrices of the same dimension is defined as $(A: B):=\sum_{i} \sum_{j} a_{i j} b_{i j}$ and the $L^{2}$-scalarproduct is given by $(A, B):=\int_{\Omega} A: B d x$ for two matrices $A, B$ of the same dimension.

To allow for a weak problem formulation, we subdivide the interval $I$ into incremental steps $0=t_{0}<$ $t_{1}<\ldots<t_{N}=T$. In each time step, we define approximations $\left(u^{n}, \varphi^{n}\right) \approx\left(u\left(t_{n}\right), \varphi\left(t_{n}\right)\right)$ and the ir-

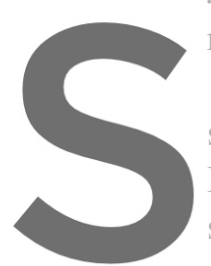
reversibility condition $\mathcal{W}:=H^{1}(\Omega)$ with a $c d$ spaces $V:=\left(H_{0}^{1}(\Omega)\right)^{2}$ In the following, the cri system of equations, a tion parameter $\kappa>0$. The stress tens
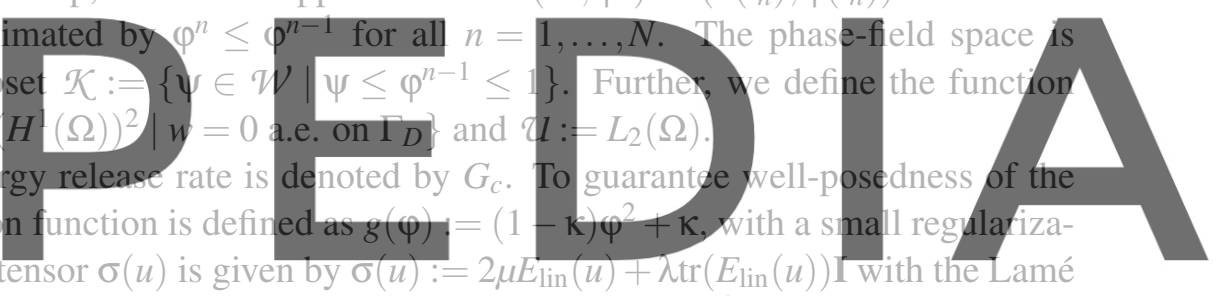

coefficients $\mu_{0} \lambda>0$. The linearized strain tensor therein is defined as $E_{\operatorname{lin}}(u):=\frac{1}{2}\left(\nabla u+\nabla u^{T}\right)$. By $\mathbf{I}$, the

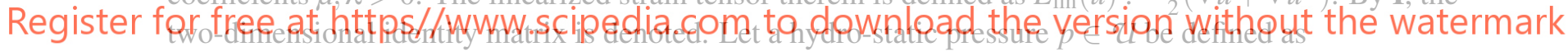

$$
p:=\lambda \operatorname{tr}\left(E_{\operatorname{lin}}(u)\right)
$$

such that the pure elasticity equation in weak form reads as:

Assume $\varphi \in \mathcal{K}$ to be given. Find $u \in \mathcal{V}$ and $p \in \mathcal{U}$ such that

$$
\begin{aligned}
g(\varphi) 2 \mu\left(E_{\operatorname{lin}}(u), E_{\operatorname{lin}}(w)\right)+g(\varphi)(\nabla \cdot w, p)=0 & \forall w \in \mathcal{V}, \\
g(\varphi)(\nabla \cdot u, q)-\frac{1}{\lambda}(p, q)=0 & \forall q \in \mathcal{U} .
\end{aligned}
$$

Remark. To avoid non-physical pressure values in the inner fracture zone, the degradation term $g(\varphi)$ is added as a coefficient in the first term of Equation (2). This yields, that even for large $\lambda$ values, the pressure values tend to zero in the fracture, where $g(\varphi)=\kappa$ and $\kappa$ is sufficiently small. 


\subsection{Choice of Underlying Energy Functional}

Three different energy functionals and two different (and commonly used) stress splitting approaches are proposed in the following. This in turn leads to six combinations of energy functionals and splitting approaches.

Wu's Unified Energy Functional. We refer to Wu [20] for a unified phase-field fracture model with the energy functional

$$
\mathrm{Wu}: E_{\varepsilon}(u, \varphi)=\int_{\Omega} \frac{g(\varphi)}{2} \sigma(u): E_{\operatorname{lin}}(u) d x+\int_{\Omega} \frac{G_{c}}{\pi} \frac{2(1-\varphi)-(1-\varphi)^{2}}{\varepsilon} d x+\int_{\Omega} \frac{G_{c}}{\pi} \varepsilon|\nabla \varphi|^{2} d x,
$$

where $\varepsilon>0$ describes the bandwidth of the transition zone between broken and unbroken material.

\section{Ambrosio-Torterelli Functional AT2. Ambrosio and Tortorelli $[2,3]$ proposed an elliptic functional defined on Sobolev spaces to approximate the Mumford-Shah functional [17]. The key idea was to replace a sharp lower-dimensional crack with a smoothed indicator function. In 2000, Bourdin et al. [8] proposed a regularized energy functional for brittle fracture. The so-called AT2 functional (named as in [18]) is defined as}
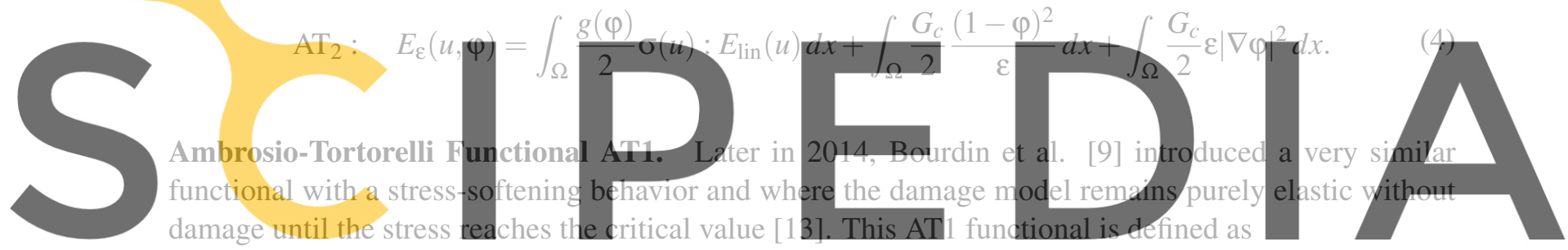

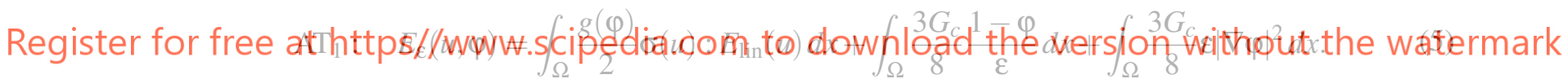

Wu's energy functional in Equation (3) uses a combination of a linear and a quadratic part in the second crack energy term, which has the advantage of a finite support for a localized phase-field [20].

\subsection{Choice of Stress Splitting Approach}

The model formulations from the previous section do not distinguish between fracture behavior in tension and compression. The most popular approaches of stress splitting are given by Miehe et al. $[15,16]$ and Amor et al. [5].

Stress splitting along to Miehe et al. As in [15, 16], we distinguish between compressive and tensile loading. This energy split is important to catch shear stresses in the material. By only applying the phase-field parameter to the tensile part of the elastic energy density, we prohibit crack propagation 
under compression [7]. For this reason, the positive part of the pressure $p^{+} \in L_{2}(\Omega)$ has to be defined as $p^{+}:=\max \{p, 0\}$, such that the tensile and compressive parts of the strain tensor can be defined as:

$$
\begin{aligned}
& \sigma^{+}(u, p)=2 \mu E_{\operatorname{lin}}^{+}(u)+p^{+} \mathbf{I}, \\
& \sigma^{-}(u, p)=2 \mu\left(E_{\operatorname{lin}}(u)-E_{\operatorname{lin}}^{+}(u)\right)+\left(p-p^{+}\right) \mathbf{I} .
\end{aligned}
$$

Remark. A detailed description on the spectral decomposition and its implementation can be find in [11, Appendix A].

Stress splitting along to Amor et al. Amor et al. [4] proposed a volumetric-deviatoric decomposition of the elastic energy density, because the regularized formulation does not distinguish between fracture behavior in tension and compression [1]:

$$
\begin{aligned}
& \sigma^{+}(u, p):=\mu \max \left\{0, \operatorname{tr}\left(E_{\operatorname{lin}}^{+}(u)\right)\right\} \mathbf{I}+2 \mu\left(E_{\operatorname{lin}}^{+}(u)-\frac{1}{3} \operatorname{tr}\left(E_{\operatorname{lin}}^{+}(u)\right) \mathbf{I}\right)+p^{+} \mathbf{I} \\
& \sigma^{-}(u, p):=\mu\left(\operatorname{tr}\left(E_{\operatorname{lin}}^{+}(u)\right)-\max \left\{0, \operatorname{tr}\left(E_{\operatorname{lin}}^{+}(u)\right)\right\}\right) \mathbf{I}+\left(p-p^{+}\right) \mathbf{I} .
\end{aligned}
$$

2.3

\section{Variational Mixed Problem Formulation}

In combination with th

incremental problem whic

Problem 1 (Mixed Phase fin

$\left\{u_{D}+v\right\}, p:=p^{n} \in \mathcal{\tau}$ a

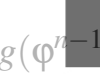

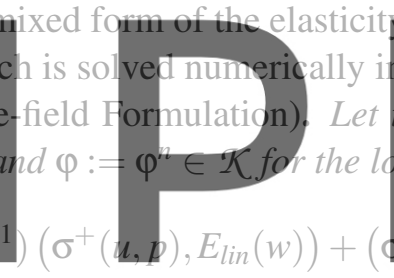
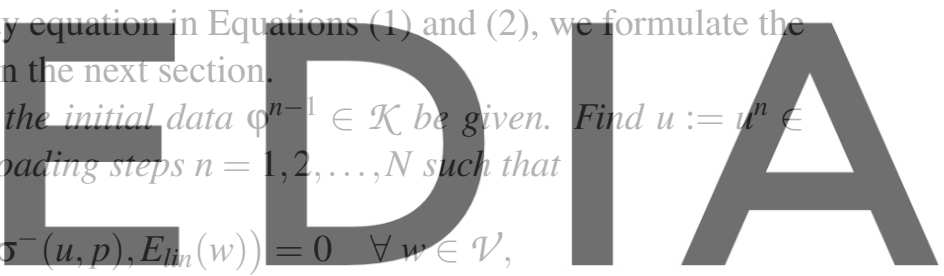

Register for free at https//www.scipedia.com to download the version without the watermark

and further, depending on the underlying energy functional, the variational inequality has to be fulfilled

Wu: $\quad(1-\kappa)\left(\varphi \sigma^{+}(u, p): E_{\text {lin }}(u), \psi-\varphi\right)+\frac{2 G_{c}}{\pi}\left(-\frac{1}{\varepsilon}(\varphi, \psi-\varphi)+\varepsilon(\nabla \varphi, \nabla(\psi-\varphi))\right) \geq 0 \quad \forall \psi \in \mathcal{K}$.

AT2: $(1-\kappa)\left(\varphi \sigma^{+}(u, p): E_{\text {lin }}(u), \psi-\varphi\right)+G_{c}\left(-\frac{1}{\varepsilon}(1-\varphi, \psi-\varphi)+\varepsilon(\nabla \varphi, \nabla(\psi-\varphi))\right) \geq 0 \quad \forall \psi \in \mathcal{K}$,

AT1: $(1-\kappa)\left(\varphi \sigma^{+}(u, p): E_{\text {lin }}(u), \psi-\varphi\right)+\left(-\frac{1}{\varepsilon}\left(\frac{3 G_{c}}{8}, \psi-\varphi\right)+\frac{3 G_{c}}{4} \varepsilon(\nabla \varphi, \nabla(\psi-\varphi))\right) \geq 0 \quad \forall \psi \in \mathcal{K}$,

Remark. In the elasticity part, time-lagging is used in the phase-field variable $\varphi$ to obtain a convex functional. See [19] for further details.

The definition of the terms $\sigma^{+}(u, p)$ and $\sigma^{-}(u, p)$ depends on the choice of the splitting approach from Section 2.2. As previously mentioned, this yields in total six different models. 


\section{EXPERIMENTS WITHIN PUNCTURED EPDM RUBBER}

Five different experiments are conducted ${ }^{1}$ four to six times for each notch height: from 6 to $18 \mathrm{~mm}$ notch height measured from the bottom boundary, punctured EPDM strips with the initial notch on the left side are stretched with a speed of $200 \mathrm{~mm} / \mathrm{s}$ until total failure. In Figure 1, the averaged crack paths of the tested puncture EPDM strips are shown.
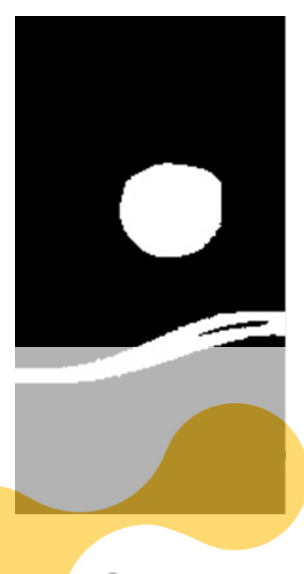

$6 \mathrm{~mm}$

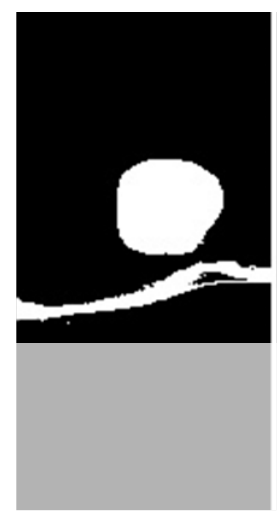

$10 \mathrm{~mm}$

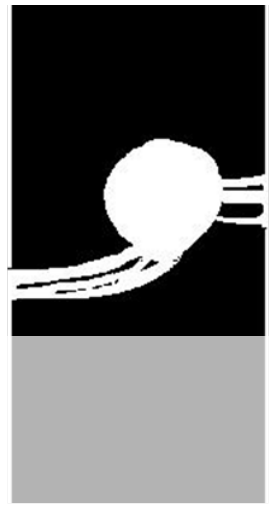

$12 \mathrm{~mm}$

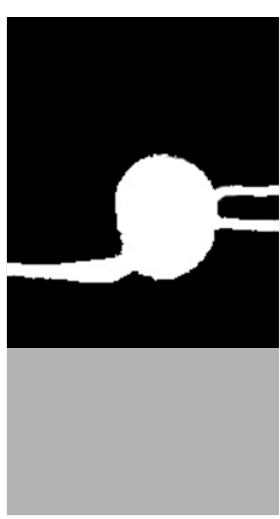

$14 \mathrm{~mm}$

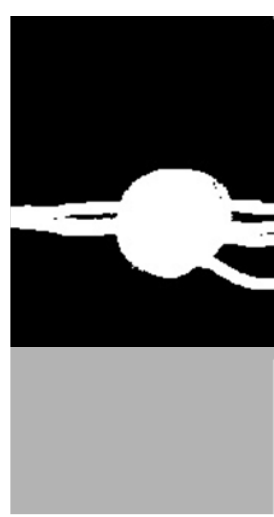

$18 \mathrm{~mm}$

Figure 1: Evaluation of the averaged crack paths (4 to 6 experiments) of the tested punctured EPDM strips with given notches at a height of 6, 10,12,14 and $18 \mathrm{~mm}$ measured from the bottom boundary (left to right). We refer the reader to [13] for more details on the experiments. In all experiments, the hole has a high impact on the the crack path is diverted towards the hole. While towards the right edge, for 12,14 , and $18 \mathrm{~mm}$ the crack is stopped for a short time by the hole, propagating

afterward nearly at the middle right inner edge of the hole towards the boundary edge of the specimen.

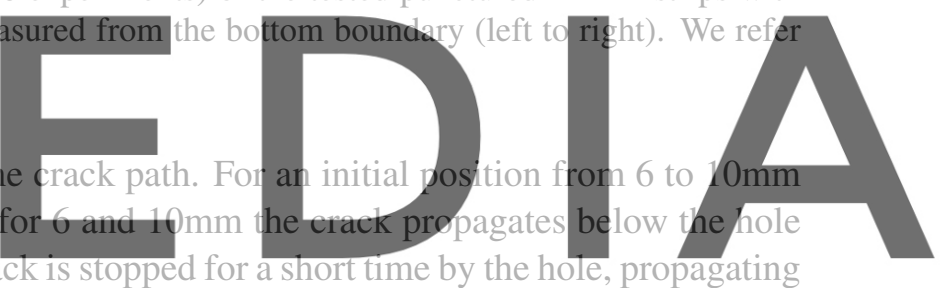

Register for free at https//www.scipedia.com to download the version without the watermark

\section{NUMERICAL RESULTS}

In comparison to the observed crack paths in the experiments from Section 3, the numerically achieved crack paths in punctured EPDM strips are discussed in the following.

\subsection{Numerical Solution}

The numerical solving of the variational phase-field fracture problem follows the approach of [10]. Therein, the crack irreversibility is enforced with a primal-dual active-set method, see [11] for further details. For the spatial discretization, we employ a Galerkin finite element method in each incremental step, where the domain $\Omega$ is partitioned into quadrilaterals. Stable Taylor-Hood elements with biquadratic shape functions $\left(Q_{2}\right)$ for the displacement field $u$ and bilinear shape functions $\left(Q_{1}\right)$ for the pressure variable $p$ and the phase-field variable $\varphi$ are used [10,14]. The implementation is embedded

\footnotetext{
${ }^{1}$ Experiments were conducted at DIK (Deutsches Institut für Kautschuktechnologie e.V.) by Nils Hendrik Kröger and Andreas Fehse. We also refer to [13].
} 
in the finite element library deal.II [6]. Further details on the underlying implementation are given in [12]. For adaptively refined meshes, a predictor-corrector scheme is used along to [11] with a threshold $\rho=0.5$ for the phase-field variable.

\subsection{Test Setup}

The geometry, the boundary conditions as well as the material and numerical parameters are given in Figure 2. In the frame of this study, we assume to have a Poisson ratio of $v=0.49$ and a critical energy release rate $G_{c}=0.97 \mathrm{~N} / \mathrm{mm}$. The elongating with a speed of $200 \mathrm{~mm} / \mathrm{s}$ is transferred in the quasi-static phase-field model with the help of an incremental step size $\delta t=10^{-3} \mathrm{~s}$. The punctured EPDM strips are fixed on the top and bottom boundary and the strips are elongated on the bottom boundary downwards.

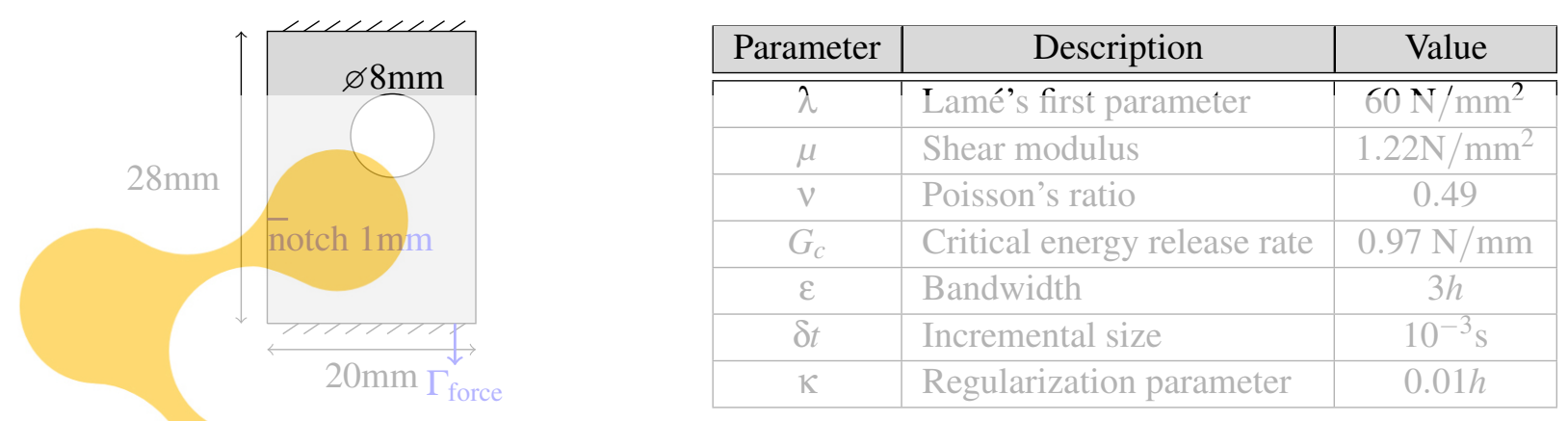

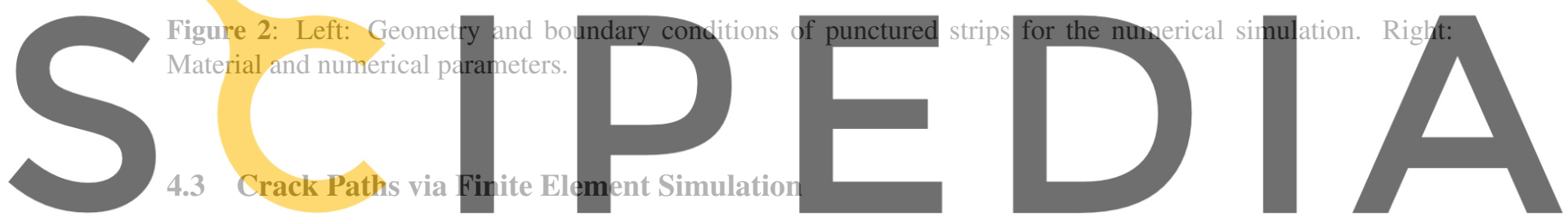

In Figures 3 to 8, the numerically achieved crack paths are presented for five different initial notch heights

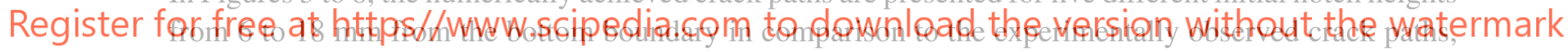
see Figure 1. Further, in the six Figures 3, 4, 5, 6, 7, and 8, the crack paths results differ due to different energy functionals from Section 2.1 and stress splitting approaches from Section 2.2.

The crack path results based on the AT2 functional from Equation (4) for both stress splitting approaches have - compared to the other energy functionals - a more smeared crack zone. In other, words, the AT2 functional with a quadratic term has a less steep gradient in the phase-field function as AT1 or Wu's approach. Aside from this, for all five notch heights in Figures 3 and 4, the crack does not start propagating from the initial crack on the left side but from the hole to the left and the right.

The crack paths in Figures 5 and 6 have a thinner zone where the phase-field variable $\varphi$ has values between 0 and 1, which means, the AT1 functional allows a sharper crack area. Anyway, aside from the third test in Figure 5 and the fourth test in Figure 6, the computed crack paths do not match the experimentally achieved crack paths from Figure 1. A high sensitivity around the circular hole is observed for all AT2 and AT1 tests.

Based on Wu's functional and Miehe splitting, the crack paths for all five test configurations look promising in Figure 7, but no convergence of the nonlinear solver within 100 Newton steps was achieved in the 

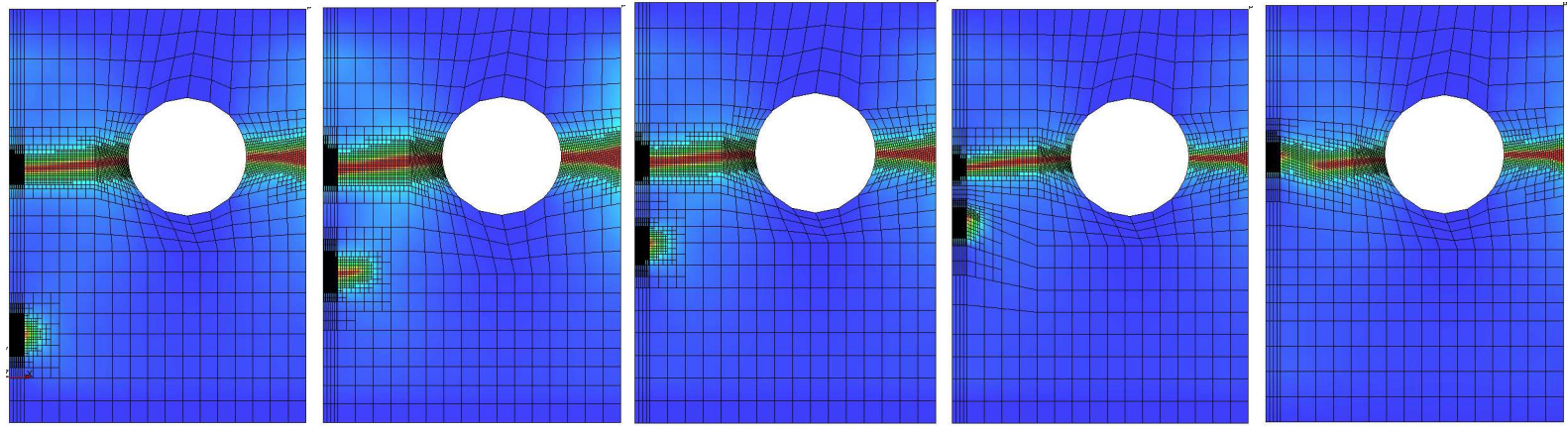

Figure 3: Crack paths in punctured EPDM strips based on AT2 functional in Equation (4) and Miehe splitting in Equation (6) with a given notch at 6,10,12,14 and $18 \mathrm{~mm}$ from left to right.
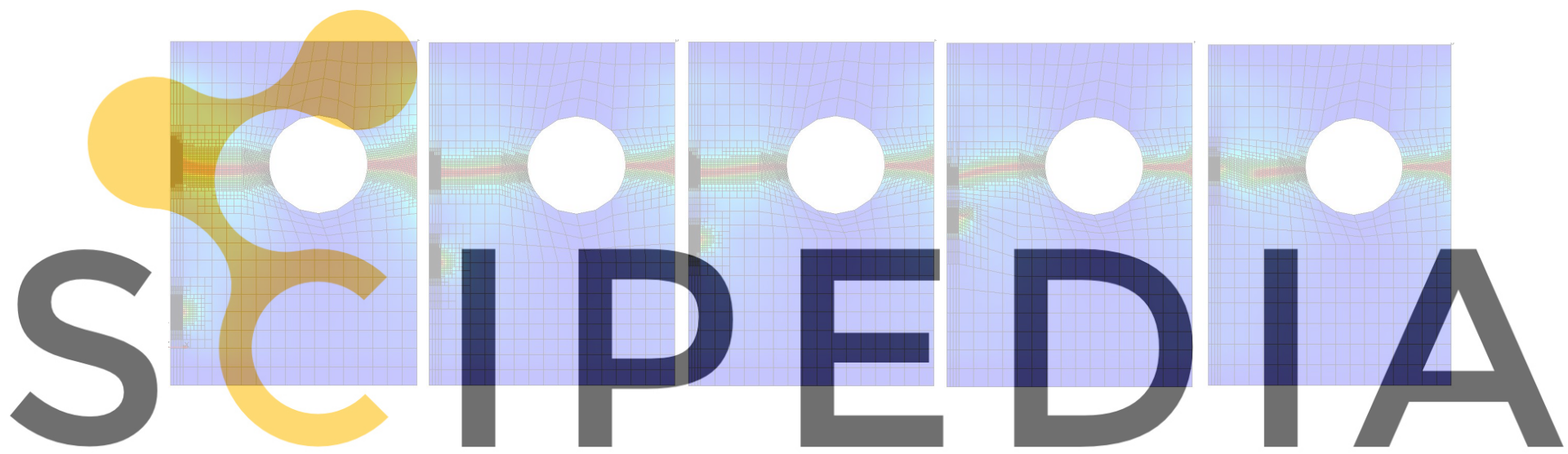

Figure 4: Crack paths in punctured EPDM strips based on AT2 functional in Equation (4) and Amor splitting in

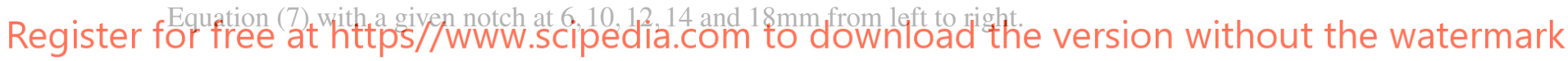
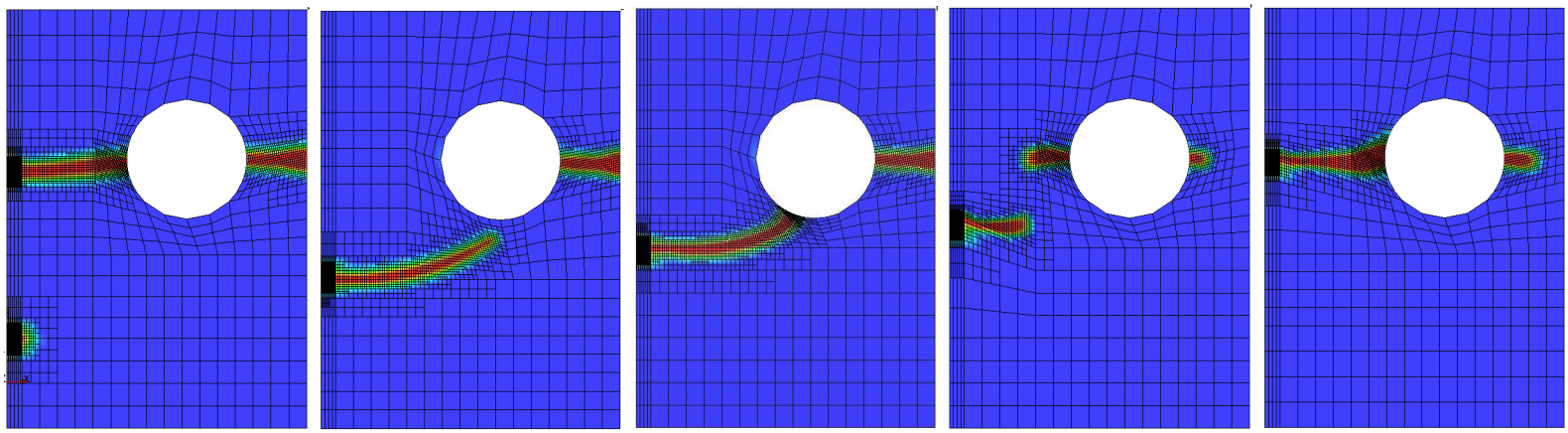

Figure 5: Crack paths in punctured EPDM strips based on AT1 functional in Equation (5) and Miehe splitting in Equation (6) with a given notch at 6,10,12,14 and $18 \mathrm{~mm}$ from left to right. 

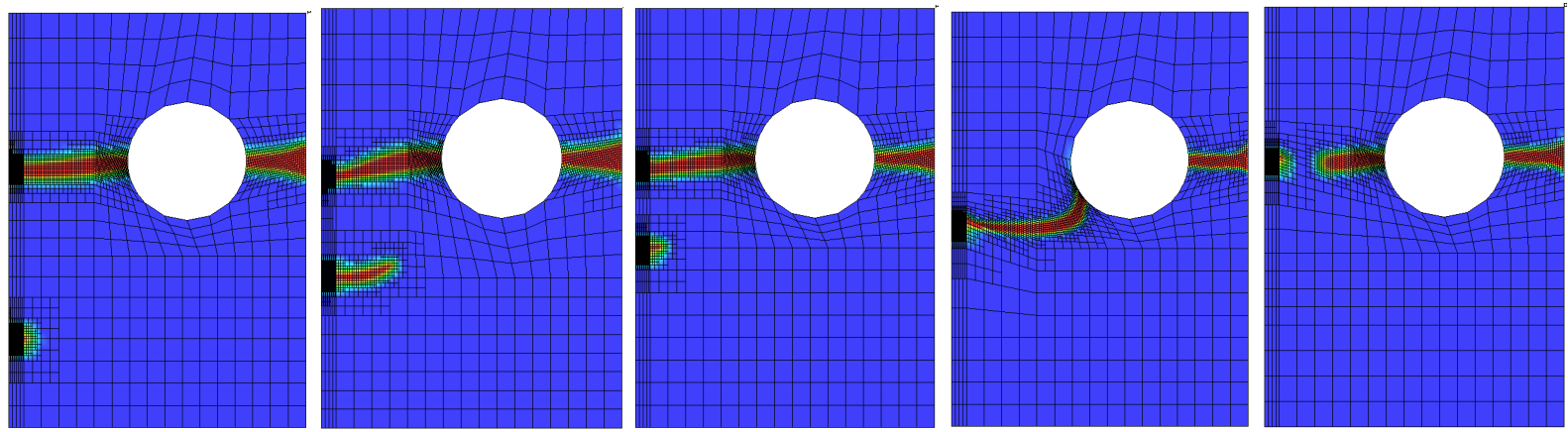

Figure 6: Crack paths in punctured EPDM strips based on AT1 functional in Equation (5) and Amor splitting in Equation (7) with a given notch at 6,10,12,14 and $18 \mathrm{~mm}$ from left to right.
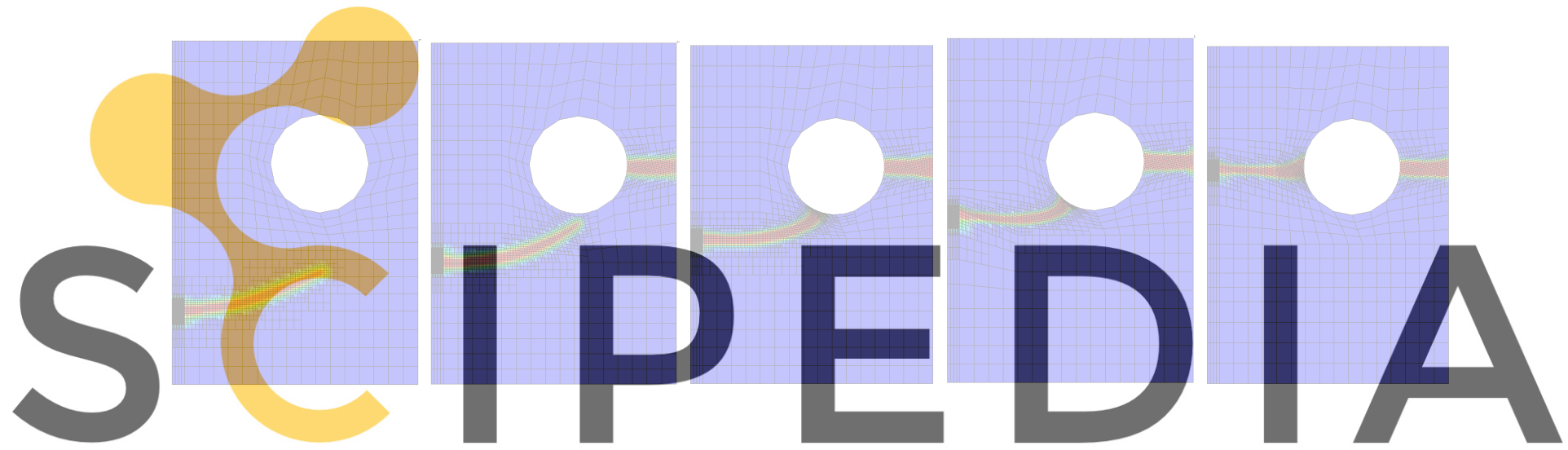

Figure 7: Crack paths in punctured EPDM strips based on Wu's energy functional in Equation (3) and Miehe Register forliting in Equation (6) with a given notgh at 6.10 12.14 and 18 mm fropthe left to right.
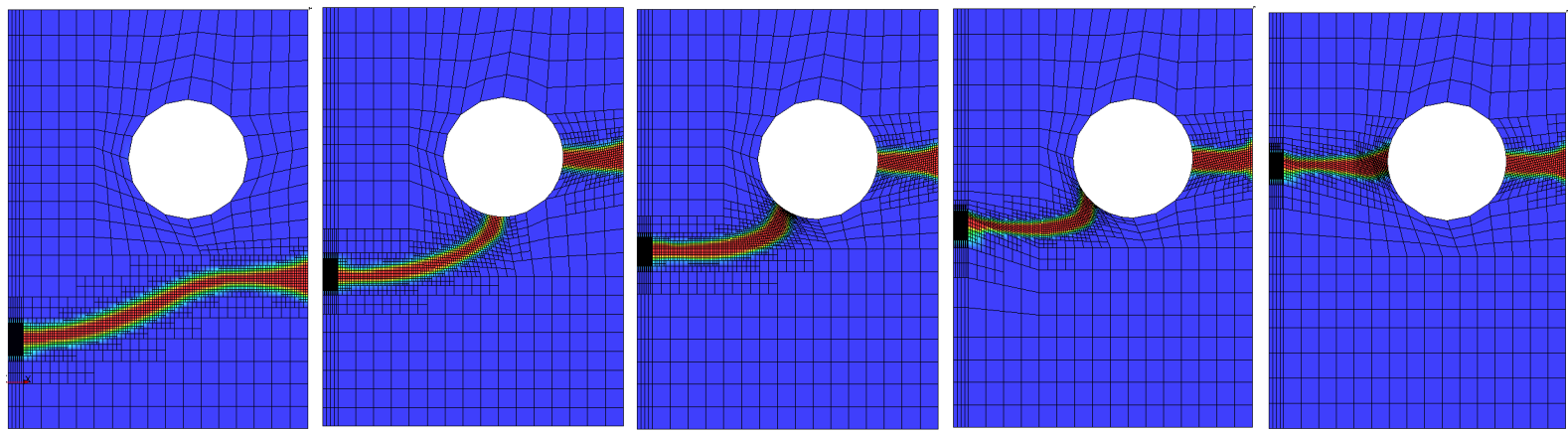

Figure 8: Crack paths in punctured EPDM strips based on Wu's energy functional in Equation (3) and Amor splitting in Equation (7) with a given notch at 6,10,12,14 and 18mm from left to right. 
first two tests with a notch height of 6 and $10 \mathrm{~mm}$ at the time point of the displayed snapshots. A reason for this could be the observed sensitivity on the incremental step size or the mesh size around the inclusion. In Figure 8, the crack paths for all five test cases look very similar to the experimentally observed crack paths. It can be inferred that the Wu functional with Amor splitting fits best to this experimental setup and possibly more general to configurations with holes as already mentioned in [21].

\section{CONCLUSIONS}

Phase-field fracture modeling in mixed form can be used to simulate cracks in nearly incompressible materials [14]. The work on hand focuses on the crack paths in punctured EPDM strips where the underlying phase-field fracture model differs in terms of three different energy functional definitions (Ambrosio-Tortorelli functionals AT2 and AT1 and the model of Wu [20]) and two different stress splitting approach (Miehe et al. [15] and Amor et al. [4]). In total, six possible combinations are used for tests in punctured EPDM strips. The crack path profiles of Wu's functional and Amor's stress splitting look most promising compared to the experimentally achieved crack paths for all five test cases. In future work, further quantities of interest such as crack energy, bulk energy, and load-displacement curves will be compared for all six presented model problem formulations.

\section{ACKNOWLEDGMENT}

The authors would like to thank Nils Hendrik Kröger and Andreas Fehse for conducting experiments at the DIK. Further, the work has been supported by the DFG, SPP 1748 (392587580, WI 4367/2-1).

REFERENCES

[1] Marreddy Ambati, Tymofiy Gerasimov, and
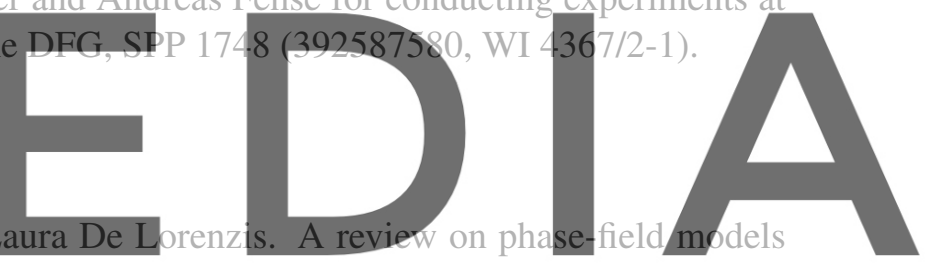

of brittle fracture and a new fast hybrid formulation. Computational Mechanics, 55(2):383-405,

Register for free 3 at https//www.scipedia.com to download the version without the watermark

[2] Luigi Ambrosio and Vincenzo Maria Tortorelli. Approximation of functional depending on jumps by elliptic functional via t-convergence. Communications on Pure and Applied Mathematics, 43(8):999-1036, 1990.

[3] Luigi Ambrosio and Vincenzo Maria Tortorelli. On the approximation of free discontinuity problems. Bollettino dell'unione matematica italiana B., 6(1):105-123, 1992.

[4] Hanen Amor, Jean-Jacques Marigo, and Corrado Maurini. Regularized formulation of the variational brittle fracture with unilateral contact: Numerical experiments. Journal of the Mechanics and Physics of Solids, 57(8):1209-1229, 2009.

[5] Hanen Amor, Jean-Jacques Marigo, and Corrado Maurini. Regularized formulation of the variational brittle fracture with unilateral contact: Numerical experiments. J. Mech. Phys. Solids, 57:1209-1229, 2009.

[6] Daniel Arndt, Wolfgang Bangerth, Bruno Blais, Thomas C Clevenger, Marc Fehling, Alexander V Grayver, Timo Heister, Luca Heltai, Martin Kronbichler, Matthias Maier, et al. The deal. ii library, version 9.2. Journal of Numerical Mathematics, 1(ahead-of-print), 2020. 
[7] Michael J Borden, Clemens V Verhoosel, Michael A Scott, Thomas JR Hughes, and Chad M Landis. A phase-field description of dynamic brittle fracture. Computer Methods in Applied Mechanics and Engineering, 217:77-95, 2012.

[8] Blaise Bourdin, Gilles A Francfort, and Jean-Jacques Marigo. Numerical experiments in revisited brittle fracture. Journal of the Mechanics and Physics of Solids, 48(4):797-826, 2000.

[9] Blaise Bourdin, Jean-Jacques Marigo, Corrado Maurini, and Paul Sicsic. Morphogenesis and propagation of complex cracks induced by thermal shocks. Physical review letters, 112(1):014301, 2014.

[10] Andreas Fehse, Nils Hendrik Kröger, Katrin Mang, and Thomas Wick. Crack path comparisons of a mixed phase-field fracture model and experiments in punctured EPDM strips. PAMM, 20(1):e202000335, 2021.

[11] Timo Heister, Mary F Wheeler, and Thomas Wick. A primal-dual active set method and predictorcorrector mesh adaptivity for computing fracture propagation using a phase-field approach. Computer Methods in Applied Mechanics and Engineering, 290:466-495, 2015.

[12] Timo Heister and Thomas Wick. pfm-cracks: A parallel-adaptive framework for phase-field fracture propagation. Software Impacts, 6:100045, 2020.

[13] Katrin Mang, Andreas Fehse, Nils Hendrik Kröger, and Thomas Wick. A mixed phase-field fracture model for crack propagation in punctured EPDM strips. submitted November, 2020.
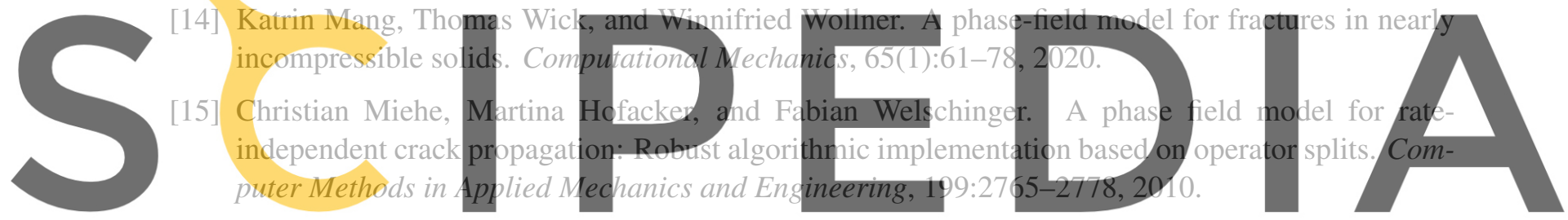

[16] Christian Miehe, Martina Hofacker, and Fabian Welschinger. Thermodynamically consistent phase-

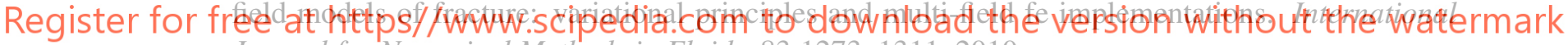
Journal for Numerical Methods in Fluids, 83:1273-1311, 2010.

[17] David Bryant Mumford and Jayant Shah. Optimal approximations by piecewise smooth functions and associated variational problems. Communications on pure and applied mathematics, 1989.

[18] Erwan Tanné, Tianyi Li, Blaise Bourdin, J-J Marigo, and Corrado Maurini. Crack nucleation in variational phase-field models of brittle fracture. Journal of the Mechanics and Physics of Solids, 110:80-99, 2018.

[19] Thomas Wick. Multiphysics Phase-Field Fracture: Modeling, Adaptive Discretizations, and Solvers, volume 28. Walter de Gruyter GmbH \& Co KG, 2020.

[20] Jian-Ying Wu. A unified phase-field theory for the mechanics of damage and quasi-brittle failure. Journal of the Mechanics and Physics of Solids, 103:72-99, 2017.

[21] Jian-Ying Wu, Vinh Phu Nguyen, Chi Thanh Nguyen, Danas Sutula, Stephane Bordas, and Sina Sinaie. Phase field modeling of fracture. Advances in applied mechancis: multi-scale theory and computation, 52, 2018. 